\title{
ALIASING FREE FOR MIXED SPECTRA FOR STABLE PROCESSES
}

\author{
Rachid Sabre \\ Biogeosciences (UMR CNRS/uB 6282), University of Burgundy, 26, Bd \\ Docteur Petitjean, Dijon, France
}

\begin{abstract}
This work focuses on the symmetric alpha stable processes with continuous time frequently used in modeling the signal with indefinitely growing variance when the spectral measure is mixed: sum of a continuous meseare and discrete measure. The objective of this paper is to estimate the spectral density of the continuous part from discrete observations of the signal. For that, we propose a method based on a sample of the signal at a periodic instant. The Jackson polynomial kernel is used for construct a periodogram. We smooth this periodogram by two spectral windows taking into account the width of the interval where the spectral density is nonzero. This technique allows to circumvent the phenomenon of aliasing often encountered in the estimation from the discrete observations of a process with a continuous time.
\end{abstract}

\section{KEYWORDS}

Spectral density, stable processes, periodogram, smoothing estimate, aliasing

\section{INTRODUCTION}

Stable alpha processes have been of interest to several research authors for their multiple applications when we have random signals with variance indefinitely increase. The harmonizable process is an important example of a symmetric $\alpha_{\text {-stable process, and its proprieties have been }}$ considered by numerous authors like [1]-[10] to name a few.

In particular, stable symmetric processes find their place in various applications and in various fields such as: physics, biology, electronics and electricity, hydrology, economies, communications and radar applications, ...ect. See: [11]-[22]. This work considers a symmetric alpha stable harmonizable process $X=\{X(t): t \in R\}$. Alternatively $X$ has the integral representation:

$$
X(t)=\int \exp [i(t \lambda)] d \xi(\lambda)
$$

where $1<\alpha<2$ and $\xi$ is a complex valued symmetric $\alpha$-stable random measure on $R$ with independent and isotropic increments. The measure defined by $m(A)=|\xi(A)|_{\alpha}^{\alpha}$ (see [4]) is called "control" measure or spectral measure." The spectral density function was already estimated in different cases: by E.Masry and S.Combanis [4] when the time of the process is continuous, by Sabre [23] when the time of the process is discrete and by R. Sabre [24]-[25] when the time of the process is p-adic. 
This work considers a general case where we suppose that the spectral measure is the sum of an absolutely continuous measure with respect to Lebesgue measure and a discrete measure:

$$
d \mu(\lambda)=\phi(x) d x+\sum_{i=1}^{q} c_{i} \delta_{w_{i}}
$$

where $\delta$ is a Dirac measure, $\phi$ is nonnegative integrable and bounded function. $c_{i}$ is an unknown positive real number and $w_{i}$ is an unknown real number. Assume that $w_{i} \neq 0$. The function $\phi$ is called the spectral density. Discrete measure is due to random repeated value jumps during experimental measurements. The spectral density $\phi$ represents the distribution of the energy carried by the signal.

Our goal is to establish a non-parametric estimate of the spectral density $\phi$ from discrete observations of $X(t)$. This is motived by the fact that, in practice, it is not obvious to observe the process on continuous interval of time. Indeed, we sampled the process at instants $t_{n}$, equally distant, i.e., $t_{n}=n \tau, \tau>0$. It is known that aliasing of $\phi$ occurs. For more details about aliasing phenomenon, see [26]. To avoid this difficulty, we suppose that the spectral density $\phi$ is vanishing for $|\lambda|>\Omega$ where $\Omega$ is a nonnegative real number. From some smoothing, we construct an estimate depending on $\Omega$ and we show that it is asymptotically unbiased and consistent.

Briefly, we indicate the organisation of this paper: the outline in this paper is as follows: we present in the second section two technical lemmas, the preiodogram and show that this periodogram is asymptotically unbiased estimated but not consistent. In the third section, we smooth this periodogram by two chosen spectral windows to estimate the spectral density at jump points. We show that the smoothing periodogram is a consistent estimator.

\section{The Periodogram ANd its Proprieties}

First, we introduce some basic notations and properties of the Jackson's polynomial kernel. Let $N$ is the size of sample of $X$. Let $k$ and $n$ are the numbers satisfying: $N-1=2 k(n-1) \quad$ with $\quad n \in N \quad k \in N \cup\left\{\frac{1}{2}\right\} \quad k=\frac{1}{2} \quad$ if $\quad n=2 n_{1}-1, n_{1} \in N$.

The Jackson's polynomial kernel is defined by: $\left|H_{N}(\lambda)\right|^{\alpha}=\left|A_{N} H^{(N)}(\lambda)\right|^{\alpha}$ where

$$
H^{(N)}(\lambda)=\frac{1}{q_{k, n}}\left(\frac{\sin \frac{n \lambda}{2}}{\sin \frac{\lambda}{2}}\right)^{2 k} \text { with } \quad q_{k, n}=\frac{1}{2 \pi} \int_{-\pi}^{\pi}\left(\frac{\sin \frac{n \lambda}{2}}{\sin \frac{\lambda}{2}}\right)^{2 k} d \lambda
$$


where $A_{N}=\left(B_{\alpha, N}\right)^{\frac{-1}{\alpha}}$ with $B_{\alpha, N}=\int_{-\pi}^{\pi}\left|H^{(N)}(\lambda)\right|^{\alpha} d \lambda$.

We give the following lemmas which are used in the rest of this paper. Their proof are given in [23].

Lemma 2.1 There is a non negative function ${ }^{h_{k}}$ such as:

Lemma 2.2 Let

$$
\begin{gathered}
H^{(N)}(\lambda)=\sum_{m=-k(n-1)}^{k(n-1)} h_{k}\left(\frac{m}{n}\right) \cos (m \lambda) \\
B_{\alpha, N}^{\prime}=\int_{-\pi}^{\pi}\left|\frac{\sin \frac{n \lambda}{2}}{\sin \frac{\lambda}{2}}\right|^{2 k \alpha} d \lambda \text { and } J_{N, \alpha}=\int_{-\pi}^{\pi}|u|^{\gamma}\left|H_{N}(u)\right|^{\alpha} d u
\end{gathered}
$$

where

$\gamma \in] 0,2]$, then

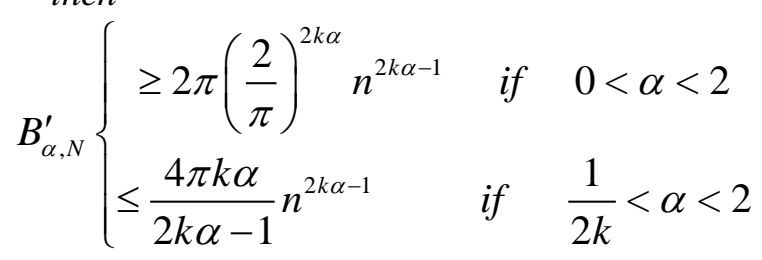

And

$$
J_{N, \alpha} \leq\left\{\begin{array}{lll}
\frac{\pi^{\gamma+2 k \alpha}}{2^{2 k \alpha}(\gamma-2 k \alpha+1)} \frac{1}{n^{2 k \alpha-1}} & \text { if } & \frac{1}{2 k}<\alpha<\frac{\gamma+1}{2 k} \\
\frac{2 k \alpha \pi^{\gamma+2 k \alpha}}{2^{2 k \alpha}(\gamma+1)(2 k \alpha-\gamma-1)} \frac{1}{n^{\gamma}} & \text { if } & \frac{\gamma+1}{2 k}<\alpha<2
\end{array}\right.
$$

In this section, we give a periodogram and we develop its proprieties. Assume that the process $X(t)$, defined in (1), is observed at instants $t_{j}=j \tau, j=1,2, \ldots N$ and $\tau=\frac{2 \pi}{\omega}$, where $\omega$ is a real number strictly greater than $2 \Omega$. We define the periodogram $\hat{I}_{N}$ on $]-\Omega, \Omega[$ as follows:

$$
\hat{I}_{N}(\lambda)=C_{p, \alpha}\left|I_{N}(\lambda)\right|^{p}, \quad 0<p<\frac{\alpha}{2}
$$

where

$$
I_{N}(\lambda)=[\tau]^{\frac{1}{\alpha}} A_{N} \operatorname{Re}\left[\sum_{n^{\prime}=-k(n-1)}^{n^{\prime}=k(n-1)} h_{k}\left(\frac{n^{\prime}}{n}\right) \exp \left\{-i\left(n^{\prime} \tau \lambda\right)\right\} X\left(n^{\prime} \tau+k(n-1) \tau\right)\right],
$$


and the normalisation constant $C_{p, \alpha}$ is given by $C_{p, \alpha}=\frac{D_{p}}{F_{p, \alpha}\left[C_{\alpha}\right]^{p / \alpha}}$, with

$$
D_{p}=\int_{-\infty}^{\infty} \frac{1-\cos (u)}{|u|^{1+p}} d u \quad \text { and } \quad F_{p, \alpha}=\int_{-\infty}^{\infty} \frac{1-e^{-|u|^{a}}}{|u|^{1+p}} d u
$$

\section{Lemma 2.3}

The characteristic function of $I_{N}(\lambda), \quad E \exp \left[\operatorname{irI}_{N}(\lambda)\right]$, converges to $\exp \left[-C_{\alpha}|r|^{\alpha}\left(\psi_{N, 1}(\lambda)+\psi_{N, 2}(\lambda)\right]\right.$. where

$$
\psi_{N, 1}(\lambda)=\int_{-\pi}^{\pi}\left|H_{N}(y-\tau \lambda)\right|^{\alpha} \phi\left(\frac{y}{\tau}\right) d y \quad \text { and } \quad \psi_{N, 2}(\lambda)=\sum_{i=1}^{q} c_{i}\left|H_{N}\left(w_{i}-\tau \lambda\right)\right|^{\alpha}
$$

\section{Proof}

By substituting (1) in the expression of $I_{N}$, we have:

$$
I_{N}(\lambda)=[\tau]^{\frac{1}{\alpha}} A_{N} \operatorname{Re} \int_{R} \sum_{n^{\prime}=-k(n-1)}^{n^{\prime}=k(n-1)} h_{k}\left(\frac{n^{\prime}}{n}\right) \exp \left\{i\left[n^{\prime} \tau(\lambda-u)\right]\right\} \exp \{i[\tau u k(n-1)]\} d \xi(u)
$$

It follows from [1] and the definition of the Jackson polynomial kernel that the characteristic function is the form:

$$
E \exp \left[i r I_{N}(\lambda)\right]=\exp \left[-C_{a}|r|^{a} \psi_{N}(\lambda)\right]
$$

where $\psi_{N}(\lambda)=\psi_{N, 1}(\lambda)+\psi_{N, 2}(\lambda) \quad$ with

$\psi_{N, 1}(\lambda)=\int_{R}\left|H_{N}(v-\tau \lambda)\right|^{a} \phi\left(\frac{v}{\tau}\right) d v \quad$ and $\quad \psi_{N, 2}(\lambda)=\sum_{i=1}^{q} c_{i}\left|H_{N}\left(w_{i}-\tau \lambda\right)\right|^{\alpha}$

We can write $\psi_{N, 1}(\lambda)=\int_{R}\left|H_{N}(v-\tau \lambda)\right|^{a} \phi\left(\frac{v}{\tau}\right) d v=\sum_{j \in Z} \int_{(2 j-1) \pi}^{(2 j+1) \pi}\left|H_{N}(v-\tau \lambda)\right|^{a} \phi\left(\frac{v}{\tau}\right) d v$.

Putting $v=y-2 \pi j$ and using the fact that $H_{N}$ is $2 \pi$-periodic, we obtain $\psi_{N}(\lambda)=\sum_{j \in Z} \int_{-\pi}^{\pi}\left|H_{N}(y-\tau \lambda)\right|^{a} \phi_{j}(y) d y,{ }_{\text {where }} \phi_{j}(y)=\phi\left(\frac{y}{\tau}-\frac{2 \pi}{\tau} j\right) .{ }_{\text {Let }} j$ be an integer 
such that $-\Omega<\frac{y-2 \pi j}{\tau}<\Omega$. Using the fact that $\tau \Omega<\pi$ and $|y|<\pi$, we get $|j|<\frac{\tau \Omega}{2 \pi}+\frac{1}{2}<1$ and then $j=0$. Consequently:

$$
\psi_{N, 1}(\lambda)=\int_{-\pi}^{\pi}\left|H_{N}(y-\tau \lambda)\right|^{\alpha} \phi\left(\frac{y}{\tau}\right) d y
$$

Theorem 2.4 Let $-\Omega<\lambda<\Omega$ then $E\left[\hat{I}_{N}(\lambda)\right]=\left[\psi_{N}(\lambda)\right]^{\frac{p}{\alpha}}$,

\section{Proof}

As in [4], we use the following equality: for all real $x$ and $0<p<2$,

$$
|x|^{p}=D_{p}^{-1} \int_{-\infty}^{\infty} \frac{1-\cos (x u)}{|u|^{1+p}} d u=D_{p}^{-1} \operatorname{Re} \int_{-\infty}^{\infty} \frac{1-e^{i x u}}{|u|^{1+p}} d u,
$$

replacing $x$ by $I_{N}$, we obtain

$$
\hat{I}_{N}(\lambda)=\frac{1}{F_{p, \alpha}\left[C_{a}\right]^{p / \alpha}} \operatorname{Re} \int_{-\infty}^{\infty} \frac{1-\exp \left\{i u I_{N}(\lambda)\right\}}{|u|^{1+p}} d u,
$$

Using (6) and the definition of the $F_{p, \alpha}$, we get

$$
\begin{aligned}
E \hat{I}_{N}(\lambda) & =\frac{1}{F_{p, \alpha}\left[C_{a}\right]^{p / \alpha}} \int_{R} \frac{1-\exp \left\{-C_{a}|u|^{\alpha} \psi_{N}(\lambda)\right\}}{|u|^{1+p}} d u \\
& =\left[\psi_{N}(\lambda)\right]^{p / \alpha}
\end{aligned}
$$

\section{Smoothing Periodogram}

In order to obtain a consistent estimate of $[\phi(\lambda)]^{\frac{p}{\alpha}}$, we smooth the periodogram via spectral windows depending on whether $\tau \lambda$ is a jump point or not $\left(\tau \lambda \neq w_{i}\right)$.

$$
f_{N}(\lambda)=\left\{\begin{array}{cc}
f_{N}^{(1)}(\lambda) \text { if } \tau \lambda \notin\left\{w_{1}, w_{2}, \cdots, w_{q}\right\} \\
\frac{f_{N}^{(2)}(\lambda)-c f_{N}^{(1)}(\lambda)}{1-c} & \text { else }
\end{array}\right.
$$

where $f_{N}^{(1)}(\lambda)=\int_{-\pi}^{\pi} W_{N}^{(1)}(\lambda-u) \hat{I}_{N}(u) d u$ and $f_{N}^{(2)}(\lambda)=\int_{-\pi}^{\pi} W_{N}^{(2)}(\lambda-u) \hat{I}_{N}(u) d u$ 
The spectral windows $W_{N}^{(1)}$ and $W_{N}^{(2)}$ are defined by: $W_{N}^{(1)}(x)=M_{N}^{(1)} W\left(M_{N}^{(1)} x\right)$ and $W_{N}^{(2)}(x)=M_{N}^{(2))} W\left(M_{N}^{(2)} x\right)$ with $W$ is an even nonnegative, continuous function, vanishing for $|\lambda|>1$ such that $\int_{-1}^{1} W(u) d u=1$. The bandwidths $\left.M_{N}^{(1)}\right)$ and $\left.M_{N}^{(2)}\right)$ satisfying: $c=\frac{M_{N}^{(2)}}{M_{N}^{(1)}}$ $\lim _{N \rightarrow \infty} M_{N}^{(i)}=+\infty, \quad \lim _{N \rightarrow \infty} \frac{M_{N}^{(i)}}{N}=0$ for $i=1,2, \quad \lim _{N \rightarrow+\infty} \frac{M_{N}^{(2)}}{M_{N}^{(1)}}=0$ and such that $\left.W\left(M_{N}^{(2)} \theta\right)=W\left(M_{N}^{(1)} \theta\right) \forall \theta \in\right]-\frac{1}{M_{N}^{(1)}}, \frac{1}{M_{N}^{(1)}}[$.

We first show that $f_{N}(\lambda)$ is an asymptotically unbiased estimator of $[\phi(\lambda)]^{\frac{p}{\alpha}}$ for $-\Omega<\lambda<\Omega$ and $\tau \lambda \notin\left\{w_{1}, w_{2}, \cdots, w_{q}\right\}$.

\section{Theorem 3.1}

Let $-\Omega<\lambda<\Omega$, such that $\tau \lambda \notin\left\{w_{1}, w_{2}, \cdots, w_{q}\right\}$. Then, $E\left[f_{N}(\lambda)\right]-[\phi(\lambda)]^{\frac{p}{\alpha}}=\mathrm{o}(1)$. If $\phi$ satisfies the hypothesis $|\phi(x)-\phi(y)| \leq c s t e|(x-y)|^{-\gamma}$, with $\gamma<2 k \alpha-1$, then,

$$
E\left[f_{N}(\lambda)\right]-[\phi(\lambda)]^{\frac{p}{\alpha}}=\left\{\begin{array}{c}
O\left(\frac{1}{n^{(2 k \alpha-1)}}+\frac{1}{M_{N}^{(1) \gamma}}\right) \quad \text { if } \quad \lambda \neq 0 \\
O\left(\frac{1}{M_{N}^{(1)} n^{(2 k \alpha-1)}}+\frac{1}{M_{N}^{(1) \gamma}}+\frac{1}{n^{2 k \alpha-1}}\right) \quad \text { if } \quad \lambda=0
\end{array} .\right.
$$

\section{Proof}

By the definition of the spectral window, we have:

$$
E\left[f_{N}(\lambda)\right]=\int_{R} M_{N}^{(1)} W\left[M_{N}^{(1)}(\lambda-u)\right] E\left[\hat{I}_{N}(u)\right] d u .
$$

Let $M_{N}^{(1)}(\lambda-u)=v$ and from (11), we obtain:

$$
E\left[f_{N}(\lambda)\right]=\int_{-1}^{1} W(v)\left[\psi_{N}\left(\lambda-\frac{v}{M_{N}^{(1)}}\right)\right]^{\frac{p}{\alpha}} d v
$$

Using the fact that $\int_{-1}^{1} W(u) d u=1$ and the inequality (4), we get: 


$$
\left|E\left[f_{N}(\lambda)\right]-[\phi(\lambda)]^{\frac{p}{\alpha}}\right| \leq \int_{-1}^{1} W(v)\left|\psi_{N}\left(\lambda-\frac{v}{M_{N}^{(1)}}\right)-\phi(\lambda)\right|^{\frac{p}{\alpha}} d v
$$

Since $\frac{p}{\alpha}<1$, we obtain

$$
\left|\psi_{N}\left(\lambda-\frac{v}{M_{N}^{(1)}}\right)-\phi(\lambda)\right|^{\frac{p}{\alpha}} \leq\left|\psi_{N, 1}\left(\lambda-\frac{v}{M_{N}^{(1)}}\right)-\phi(\lambda)\right|^{\frac{p}{\alpha}}+\left|\psi_{N, 2}\left(\lambda-\frac{v}{M_{N}^{(1)}}\right)\right|^{\frac{p}{\alpha}}
$$

We now examine the limit of $\psi_{N, 1}\left(\lambda-\frac{v}{M_{N}^{(1)}}\right)$. From (3) we get:

$$
\begin{aligned}
& \psi_{N, 1}\left(\lambda-\frac{v}{M_{N}^{(1)}}\right)=\int_{-\infty}^{\infty} \mid H_{N}\left(u-\left.\tau\left(\lambda-\frac{v}{M_{N}^{(1)}}\right)||\right|^{\alpha} \phi\left(\frac{u}{\tau}\right) d u\right. \\
& \text { Let } u-\tau\left(\lambda-\frac{v}{M_{N}^{(1)}}\right)=y, \text { we obtain: } \\
& \begin{aligned}
\psi_{N, 1}\left(\lambda-\frac{v}{M_{N}^{(1)}}\right) & =\int_{R}\left|H_{N}(y)\right|^{\alpha} \phi\left(\lambda-\frac{v}{M_{N}^{(1)}}+\frac{y}{\tau}\right) d y \\
& =\sum_{j \in Z} \int_{(2 j-1) \pi}^{(2 j+1) \pi}\left|H_{N}(y)\right|^{\alpha} \phi\left(\lambda-\frac{v}{M_{N}^{(1)}}+\frac{y}{\tau}\right) d y
\end{aligned}
\end{aligned}
$$

Let $y-2 j \pi=s$. Since $\left|H_{N}(.)\right|^{\alpha}$ is $2 \pi-$ periodic function, we get

$$
\psi_{N, 1}\left(\lambda-\frac{v}{M_{N}^{(1)}}\right)=\sum_{j \in Z} \int_{-\pi}^{+\pi}\left|H_{N}(s)\right|^{\alpha} \phi\left(\lambda-\frac{v}{M_{N}^{(1)}}+\frac{s}{\tau}+\frac{2 \pi}{\tau} j\right) d s
$$

Since the function $\phi$ is uniformly continuous on $[-\Omega, \Omega]$ and the fact that $\left|H_{N}\right|^{\alpha}$ is a kernel, the right hand side of the last equality converges to $\sum_{j \in Z} \phi\left(\lambda+\frac{2 \pi j}{\tau}\right) \cdot{ }_{\text {Let }} j$ be an integer such that $-\Omega<\frac{\tau \lambda+2 \pi j}{\tau}<\Omega$. The definition of $\tau$ implies that $|\tau \lambda|<|\tau \Omega|<\pi$. It is easy to 
see that $|j|<1$ and then $j=0$. Since $H_{N}^{(1)}$ is a kernel, we obtain that $\psi_{N, 1}\left(\lambda-\frac{v}{M_{N}^{(1)}}\right)$ converges to $\phi(\lambda)$. On the other hand, $\psi_{N, 2}\left(\lambda-\frac{v}{M_{N}^{(1)}}\right)=\sum_{i=1}^{q}\left|H_{N}^{(1)}\left(w_{i}-\tau\left(\lambda-\frac{v}{M_{N}^{(1)}}\right)\right)\right|$

$$
\psi_{N, 2}\left(\lambda-\frac{v}{M_{N}^{(1)}}\right)=\sum_{i=1}^{q}\left|H_{N}^{(1)}\left(w_{i}-\tau\left(\lambda-\frac{v}{M_{N}^{(1)}}\right)\right)\right|^{\alpha}
$$

Since $\quad w_{i}$ is different from $\tau \lambda$ and from the lemma 2.2, we get $\psi_{N, 2}\left(\lambda-\frac{v}{M_{N}^{(1)}}\right) \leq\left[2 \pi\left(\frac{2}{\pi}\right)^{2 k \alpha} n^{2 k \alpha-1}\right]^{-1} \frac{1}{c t e} \sum_{i=1}^{q} c_{i}$, where cte $=\inf \left|\sin \left(\frac{w_{i}-\tau\left(\lambda-\frac{v}{M_{N}^{(1)}}\right)}{2}\right)\right|^{\alpha}$.

Therefore, $\quad \psi_{N, 2}\left(\lambda-\frac{v}{M_{N}^{(1)}}\right)=O\left(\frac{1}{n^{2 k \alpha-1}}\right)$ Thus, we have $E\left[f_{N}(\lambda)\right]-[\phi(\lambda)]^{\frac{p}{\alpha}}=o(1)$

The rate of convergence: We assume that the spectral density $\phi$ satisfies the hypothesis $H$. We denote by $F=\left|\operatorname{Bias}\left(f_{N}(\lambda)\right)\right|=\left|E\left[f_{N}(\lambda)\right]-[\phi(\lambda)]^{p / \alpha}\right|$. It follows that

$$
F \leq \frac{p}{2 \alpha} \int_{-1}^{1} W(v)\left[\left[\psi_{N}\left(\lambda-\frac{v}{M_{N}^{(1)}}\right)\right]^{\frac{p}{\alpha}-1}+[\phi(\lambda)]^{\frac{p}{\alpha}-1}\right]\left|\psi_{N}\left(\lambda-\frac{v}{M_{N}^{(1)}}\right)-\phi(\lambda)\right| d v
$$

Since $\psi_{N}\left(\lambda-\frac{v}{M_{N}}\right)$ converges to $\phi(\lambda)$, getting the rate of the convergence for $F$ requires to examine the rate of convergence of

$$
\begin{aligned}
& \int_{-1}^{1} W(v) \mid \psi_{N}\left(\lambda-\frac{v}{M_{N}^{(1)}}\right)-\phi \\
& \left.-\tau \lambda+\frac{\tau v}{M_{N}^{(1)}}\right)\left.\right|^{\alpha} \phi\left(\frac{y}{\tau}\right) d y
\end{aligned}
$$

obtain

$$
\psi_{N}\left(\lambda-\frac{v}{M_{N}^{(1)}}\right)=\left.\int_{-\pi}^{\pi}\left|H_{N}\left(y-\tau \lambda+\frac{\tau \nu}{M_{N}^{(1)}}\right)\right|\right|^{\alpha} \phi\left(\frac{y}{\tau}\right) d y
$$

Denote by $\Delta\left(\psi_{N}, \phi\right)=\psi_{N}\left(\lambda-\frac{v}{M_{N}^{(1)}}\right)-\phi(\lambda) \quad t=-\left(y-\tau \lambda+\frac{\tau v}{M_{N}^{(1)}}\right)$ and using the

condition $H$, we get

$$
\left|\Delta\left(\psi_{N}, \phi\right)\right| \leq C_{1} \int_{\tau \lambda-\frac{\tau \nu}{M_{N}}}^{\frac{\tau-\frac{\tau v}{M_{N}^{(1)}}}{M_{N}}+\pi}\left|H_{N}(t)\right|^{\alpha}\left|\frac{v}{M_{N}^{(1)}}+\frac{t}{\tau}\right|^{\gamma} d t
$$

It is easy to show that 


$$
\begin{aligned}
\int_{-1}^{1} W(v)\left|\Delta\left(\psi_{N}, \phi\right)\right| d v & \leq 2^{\gamma} C_{1}\left|\frac{1}{M_{N}^{(1)}}\right|^{\gamma} \int_{-1}^{1} W(v)|v|^{\gamma} d v \\
& +2^{\gamma} \frac{C_{1}}{\tau^{\gamma}} \int_{-1}^{1} W(v) \int_{\tau_{1} \lambda-\frac{\tau_{N}}{M_{N}^{(1)}}-\pi}^{\frac{M_{1} v}{(1)}+\pi}\left|H_{N}(t)\right|^{\alpha}|t|^{\gamma} d t d v
\end{aligned}
$$

The second integral of the right hand side is bounded as follows:

$$
\begin{aligned}
& \int_{\tau \lambda-\frac{\tau v}{M_{N}^{(1)}}-\pi}^{\tau \lambda-\frac{\tau v}{M_{N}^{(1)}+\pi}}\left|H_{N}(t)\right|^{\alpha}|t|^{\gamma} d t \leq \int_{-|\tau \lambda|-\left|\frac{\tau v}{M_{N}^{(1)}}\right|-\pi}^{-\pi}\left|H_{N}(t)\right|^{\alpha}|t|^{\gamma} d t \\
& +\quad \int_{-\pi}^{\pi}\left|H_{N}(t)\right|^{\alpha}|t|^{\gamma} d t \\
& +\int_{\pi}^{|\tau \lambda|+\left|\frac{\tau v}{M_{N}^{(1)}}\right|+\pi}\left|H_{N}(t)\right|^{\alpha}|t|^{\gamma} d t \text {. }
\end{aligned}
$$

The function $\left|H_{N}().\right|$ is even, then the first and the last integrals in the right hand side of (9) are equal. Since $\frac{\tau \nu}{M_{N}}$ converges to zero and $\tau \lambda<\tau \Omega<\pi$, for a large $N$ we have:

$$
\begin{aligned}
\int_{\pi}^{|\tau \lambda|+\left|\frac{\tau v}{M_{N}^{(1)}}\right|+\pi}\left|H_{N}(t)\right|^{\alpha}|t|^{\gamma} d t & \leq(2 \pi)^{\gamma} \int_{\pi}^{|\tau \lambda|+\left|\frac{\tau v}{M_{N}^{(1)}}\right|+\pi}\left|H_{N}(t)\right|^{\alpha} d t \\
& \leq \frac{(2 \pi)^{\gamma}}{B_{\alpha, N}^{\prime}} \frac{|\tau \lambda|+\frac{\tau}{M_{N}^{(1)}}}{\left.\sin \left(\pi+|\tau \lambda|+\frac{\tau}{M_{N}^{(1)}}\right)\right|^{2 k \alpha}}
\end{aligned}
$$

From the lemma 2.1, we obtain,

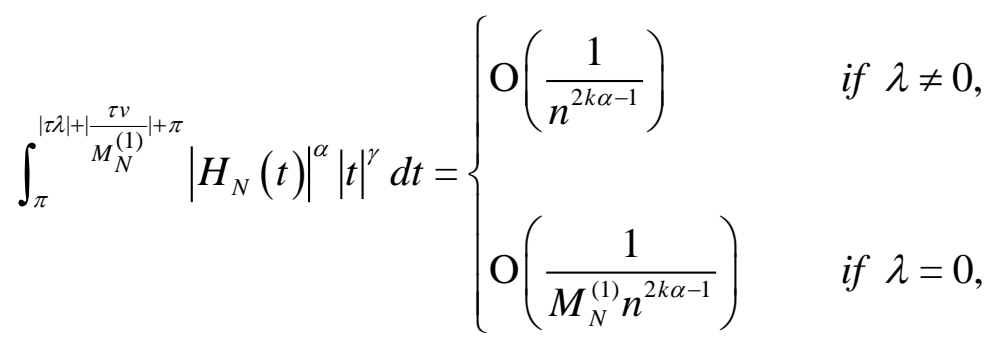

Thus, the result follows. 
Theorem 3.2. Let $\lambda$ a real nuber belonging to $]-\Omega, \Omega\left[\right.$, and $\tau \lambda=w_{i}$. Choose $k$ such that $\lim _{N \rightarrow \infty} \frac{\left(M_{N}^{(1)}\right)^{2 k \alpha}}{n^{2 k \alpha-1}}=0$. Then,

i) $\quad E\left[f_{N}(\lambda)\right]-[\phi(\lambda)]^{\frac{p}{\alpha}}=o(1)$

ii) If $\phi$ satisfiies the hypothesis $|\varphi(x)-\varphi(y)| \leq c s t e|(x-y)|^{-\gamma}$, with $\frac{\gamma+1}{2 k}<\alpha<2$, then

$$
\left|E\left[f_{N}(\lambda)\right]-[\phi(\lambda)]^{\frac{p}{\alpha}}\right|= \begin{cases}O\left(\frac{1}{\left(M_{N}^{(2)}\right)^{\gamma}}+\frac{\left(M_{N}^{(1)}\right)^{2 k \alpha}}{n^{2 k \alpha-1}}\right) \quad \text { if } & 0<\gamma \leq 1 \\ O\left(\frac{1}{M_{N}^{(2)}}+\frac{\left(M_{N}^{(1)}\right)^{2 k \alpha}}{n^{2 k \alpha-1}}\right) \quad \text { if } & 1<\gamma \leq 2\end{cases}
$$

\section{Proof :}

From the defintion of the estimator, we have

$$
\begin{gathered}
E\left[f_{N}(\lambda)\right]=\int_{-\pi}^{\pi} \frac{W_{N}^{(2)}(\lambda-u)-\frac{M_{N}^{(2)}}{M_{N}^{(1)}} W_{N}^{(1)}(\lambda-u)}{1-\frac{M_{N}^{(2)}}{M_{N}^{(1)}}}\left[\psi_{N}(u)\right]^{\underline{p}} d u \\
E\left[f_{N}(\lambda)\right]=\int_{-\pi}^{\lambda-\frac{1}{M_{N}^{(1)}}+\int_{\lambda-\frac{1}{M_{N}^{(1)}}}^{\lambda+\frac{1}{M_{N}^{(1)}}}+\int_{\lambda+\frac{1}{M_{N}^{(1)}}}^{\pi}}=E_{1}+E_{2}+E_{3} \\
\lambda-u=v, E_{2}=\int_{-\frac{1}{M_{N}^{(1)}}}^{\frac{1}{M_{N}^{(1)}}} \frac{M_{N}^{(2)}\left[W\left[M_{n}^{(2)} v\right]-W\left[M_{N}^{(1)} v\right]\right]}{1-\frac{M_{N}^{(2)}}{M_{N}^{(1)}}}\left[\psi_{N}(\lambda-v)\right]^{\frac{p}{\alpha}} d v . \\
\text { for a large N. Therefore } E_{2}=0
\end{gathered}
$$




$$
\begin{aligned}
E_{1}= & \frac{M_{N}^{(2)}}{1-\frac{M_{N}^{(2)}}{M_{N}^{(1)}}} \int_{-\pi}^{\lambda-\frac{1}{M_{N}^{(1)}}} W\left[M_{N}^{(2)}(\lambda-u)\right]\left[\psi_{N}(u)\right]^{\frac{p}{\alpha}} d u- \\
& \frac{M_{N}^{(2)}}{1-\frac{M_{N}^{(2)}}{M_{N}^{(1)}}} \int_{-\pi}^{\lambda-\frac{1}{M_{N}^{(1)}}} W\left[M_{N}^{(1)}(\lambda-u)\right]\left[\psi_{N}(u)\right]^{\frac{p}{\alpha}} d u
\end{aligned}
$$

Put $M_{N}^{(2)}(\lambda-u)=v$ in the first integral and put $M_{N}^{(1)}(\lambda-u)=w$, in the second integral and for a large $N$, we have $M_{N}^{(1)}(\lambda+\pi)>1$ et $M_{N}^{(2)}(\lambda+\pi)>1$. As $W$ is null outside of ${ }^{[-1,1]}$, for large $N$, the second integral of $E_{1}$ is zero. Therefore,

$$
E_{1}=\frac{1}{1-\frac{M_{N}^{(2)}}{M_{N}^{(1)}}} \int_{\frac{M_{N}^{(2)}}{M_{N}^{(1)}}}^{1} W(v)\left[\psi_{N}\left(\lambda-\frac{v}{M_{N}^{(2)}}\right)\right]^{\frac{p}{\alpha}} d v
$$

$E_{3}=\frac{M_{N}^{(2)}}{1-\frac{M_{N}^{(2)}}{M_{N}^{(1)}}} \int_{\lambda+\frac{1}{M_{N}^{(1)}}}^{\pi} W\left[M_{N}^{(2)}(\lambda-u)\right]\left[\psi_{N}(u)\right]^{\frac{p}{\alpha}} d u-\frac{M_{N}^{(2)}}{1-\frac{M_{N}^{(2)}}{M_{N}^{(1)}}} \int_{\lambda+\frac{1}{M_{N}^{(1)}}}^{\pi} W\left[M_{N}^{(1)}(\lambda-u)\right]\left[\psi_{N}(u)\right]^{\frac{p}{\alpha}} d u$

Putting $M_{N}^{(2)}(\lambda-u)=v$ in the first integral and $M_{N}^{(1)}(\lambda-u)=w$ in the second integral, we obtain

$$
E_{3}=\frac{1}{1-\frac{M_{N}^{(2)}}{M_{N}^{(1)}}} \int_{M_{N}^{(2)}(\lambda-\pi)}^{-\frac{M_{N}^{(2)}}{M^{(1)}}} W(v)\left[\psi_{N}\left(\lambda-\frac{v}{M_{N}^{(2)}}\right)\right]^{\frac{p}{\alpha}} d v-\frac{\frac{M_{N}^{(2)}}{M_{N}^{(1)}}}{1-\frac{M_{N}^{(2)}}{M_{N}^{(1)}}} \int_{M_{N}^{(1)}(\lambda-\pi)}^{-1} W(w)\left[\psi_{N}\left(\lambda-\frac{w}{M_{N}^{(1)}}\right)\right]^{\frac{p}{\alpha}} d w
$$

For large $N$ we have, $M_{N}^{(2)}(\lambda-\pi)<-1 \quad$ and $\quad M_{N}^{(1)}(\lambda-\pi)<-1$.

$$
E_{3}=\frac{1}{1-\frac{M_{N}^{(2)}}{M_{N}^{(1)}}} \int_{\frac{M_{N}^{(2)}}{M_{N}^{(1)}}}^{1} W(v)\left[\psi_{N}\left(\lambda+\frac{v}{M_{N}^{(2)}}\right)\right]^{\frac{p}{\alpha}} d v
$$

It is easy to show that for a large $N$ 


$$
\begin{gathered}
\frac{1}{1-\frac{M_{N}^{(2)}}{M_{N}^{(1)}}} \int_{\frac{M_{N}^{(2)}}{M_{N}^{(1)}}}^{1} W(v) d v=\frac{1}{2} . \\
\left|E\left[f_{N}(\lambda)\right]-[\phi(\lambda)]^{\frac{p}{\alpha}}\right|=\left|E_{1}+E_{3}-[\phi(\lambda)]^{\frac{p}{\alpha}}\right| \leq\left|E_{1}-\frac{1}{2}[\phi(\lambda)]^{\frac{p}{\alpha}}\right|+\left|E_{3}-\frac{1}{2}[\phi(\lambda)]^{\frac{p}{\alpha}}\right|
\end{gathered}
$$

From (10) and (12), for a large $N$, we have

$$
\left|E_{1}-\frac{1}{2}[\phi(\lambda)]^{\frac{p}{\alpha}}\right| \leq \frac{1}{1-\frac{M_{N}^{(2)}}{M_{N}^{(1)}}} \int_{\frac{M_{N}^{(2)}}{M_{N}^{(1)}}}^{1} W(v)\left|\psi_{N, 1}\left(\lambda-\frac{v}{M_{N}^{(2)}}\right)+\psi_{N_{2}}\left(\lambda-\frac{v}{M_{N}^{(2)}}\right)-\phi(\lambda)\right|^{\mid \frac{p}{\alpha}} d v
$$

As $\frac{p}{\alpha}<1$, we obtain

$$
\begin{gathered}
\left|E_{1}-\frac{1}{2}[\phi(\lambda)]^{\frac{p}{\alpha}}\right| \leq \frac{1}{1-\frac{M_{N}^{(2)}}{M_{N}^{(1)}}} \int_{\frac{M_{N}^{(2)}}{M_{N}^{(1)}}}^{1} W(v)\left|\psi_{N, 1}\left(\lambda-\frac{v}{M_{N}^{(2)}}\right)-\phi(\lambda)\right|^{\frac{p}{\alpha}} d v \\
\\
+\frac{1}{1-\frac{M_{N}^{(2)}}{M_{N}^{(1)}}} \int_{\frac{M_{N}^{(2)}}{M_{N}^{(1)}}}^{1} W(v)\left|\psi_{N, 2}\left(\lambda-\frac{v}{M_{N}^{(2)}}\right)\right|^{\frac{p}{\alpha}} d v .
\end{gathered}
$$

On the other hand,

$$
\int_{\frac{M_{N}^{(2)}}{M_{N}^{(1)}}}^{1} W(v)\left|\psi_{N, 1}\left(\lambda \pm \frac{v}{M_{N}^{(2)}}\right)-\phi(\lambda)\right|^{\frac{p}{\alpha}} d v \leq \int_{0}^{1} W(v)\left|\psi_{N, 1}\left(\lambda \pm \frac{v}{M_{N}^{(2)}}\right)-\phi(\lambda)\right|^{\frac{p}{\alpha}} d v
$$

For all $\lambda$ belonging to $]-\pi, \pi\left[\psi_{N, 1}\left(\lambda \pm \frac{v}{M_{N}^{(2)}}\right)\right.$ converges to $\phi(\lambda)$, uniformly in

$$
v \in[-1,1] . \text { Therefore, } \frac{1}{1-\frac{M_{N}^{(2)}}{M_{N}^{(1)}}} \int_{\frac{M_{N}^{(2)}}{M_{N}^{(1)}}}^{1} W(v)\left|\psi_{N, 1}\left(\lambda \pm \frac{v}{M_{N}^{(2)}}\right)-\phi(\lambda)\right|^{\frac{p}{\alpha}} d v
$$

converge to zero. 
Since $\tau \lambda=w_{i}$,

$$
\psi_{N, 2}\left(\lambda_{i} \pm \frac{v}{M_{N}^{(2)}}\right) \leq \sum_{m=1}^{q} \frac{a_{m}}{B_{\alpha, N}^{\prime}} \frac{1}{\left.\sin \left[\frac{1}{2}\left(w_{i} \pm \frac{\tau v}{M_{N}^{(2)}}-w_{m}\right)\right]\right|^{2 k \alpha}}
$$

$$
+\frac{a_{i}}{B_{\alpha, N}^{\prime}}\left|\sin \left[\frac{1}{2}\left( \pm \frac{\tau v}{M_{N}^{(2)}}\right)\right]\right|^{2 k \alpha}
$$

For all $m \neq i$,

$$
\lim _{N \rightarrow+\infty} \sup _{v \in[-1,1]} \frac{1}{\left|\sin \left[\frac{1}{2}\left(w_{i} \pm \frac{\tau v}{M_{N}^{(2)}}-w_{m}\right)\right]\right|^{2 k \alpha}}=\frac{1}{\left|\sin \left[\frac{1}{2}\left(w_{i}-w_{m}\right)\right]\right|^{2 k \alpha}}
$$

Thus, for large N, we get

$$
\sum_{m=1}^{q} \frac{a_{m}}{B_{\alpha, N}^{\prime}} \frac{1}{\left.\sin \left[\frac{1}{2}\left(w_{i} \pm \frac{\tau \nu}{M_{N}^{(2)}}-w_{m}\right)\right]\right|^{2 k \alpha}} \leq\left(\varepsilon+\frac{1}{\inf _{m \in\{1,2, \cdots, q\}-\{i\}}\left|\sin \left[\frac{1}{2}\left(w_{i}-w_{m}\right)\right]\right|^{2 k \alpha}}\right) \sum_{m=1}^{q} \frac{a_{m}}{B_{\alpha, N}^{\prime}} .
$$

From the lemma 2.1, we obtain

$$
\sum_{m=1}^{q} \frac{a_{m}}{B_{\alpha, N}^{\prime}} \frac{1}{\left|\sin \left[\frac{1}{2}\left(w_{i} \pm \frac{\tau v}{M_{N}^{(2)}}-w_{m}\right)\right]\right|^{2 k \alpha}}=O\left(\frac{1}{n^{2 k \alpha-1}}\right)
$$

On the other hand, for large $N$, we have $\sup _{v \in[0,1]}\left|\frac{\tau v}{M_{N}^{(2)}}\right|=\frac{\tau}{M_{N}^{(2)}}<\pi$. Consequently

$$
\frac{a_{i}}{B_{\alpha, N}^{\prime}}\left|\sin \left[\frac{1}{2}\left( \pm \frac{\tau v}{M_{N}^{(2)}}\right)\right]\right|^{2 k \alpha} \leq \frac{a_{i}}{B_{\alpha, N}^{\prime}} \frac{\pi^{2 k \alpha}}{\left|\frac{\tau v}{M_{N}^{(2)}}\right|^{2 k \alpha}}
$$

As $\quad \frac{\tau}{M_{N}^{(1)}} \leq \frac{\tau v}{M_{N}^{(2)}} \leq \frac{\tau}{M_{N}^{(2)}}$, we obtain $B_{\alpha, N}^{\prime}\left|\sin \left[\frac{1}{2}\left( \pm \frac{\tau v}{M_{N}^{(2)}}\right)\right]\right|^{2 k \alpha} \leq \frac{a_{i}}{B_{\alpha, N}^{\prime}} \overline{\left|\frac{\tau}{M_{N}^{(1)}}\right|^{2 k \alpha}}$

The lemma 2.2 gives 


$$
\frac{a_{i}}{B_{\alpha, N}^{\prime}} \frac{1}{\sin \left[\left.\frac{1}{2}\left(+-\frac{\tau v}{M_{N}^{(2)}}\right)\right|^{2 k \alpha}\right.}=O\left(\frac{\left(M_{N}^{(1)}\right)^{2 k \alpha}}{n^{2 k \alpha-1}}\right) .
$$

Thus, we get

$$
\frac{2^{\frac{p}{\alpha}}}{1-\frac{M_{N}^{(2)}}{M_{N}^{(1)}}} \int_{\frac{M_{N}^{(2)}}{M_{N}^{(1)}}}^{1} W(v)\left|\psi_{N, 2}\left(\tau \lambda \pm \frac{\tau v}{M_{N}^{(2)}}\right)\right|^{\frac{p}{\alpha}} d v=O\left[\frac{1}{n^{(2 k \alpha-1) \frac{p}{\alpha}}}+\left(\frac{\left(M_{N}^{(1)}\right)^{2 k \alpha}}{n^{2 k \alpha-1}}\right)^{\frac{p}{\alpha}}\right]
$$

Choosing $M_{N}^{(1)}$ such that $\frac{\left(M_{N}^{(1)}\right)^{2 k \alpha}}{n^{2 k \alpha-1}}$ converges to 0 . For example $M_{N}^{(1)}=n^{b} \quad$ with $0<b<1-\frac{1}{2 k \alpha}$. Thus, $\lim _{N \rightarrow+\infty} E\left[f_{N}(\lambda)\right]-[\phi(\lambda)]^{\frac{p}{\alpha}}=0$.

Theorem 4.2 Let $-\Omega<\lambda<\Omega$ such that $\phi(\lambda)>0$. Then, $\left.\operatorname{var}\left[f_{N}(\lambda)\right)\right]$ converges to zero. If $M_{N}^{(1)}=n_{\text {with }}^{c} \frac{1}{2 k^{2} \alpha^{2}}<c<\frac{1}{2}$, then $\left.\operatorname{var}\left[f_{N}(\lambda)\right)\right]=\mathrm{O}\left(\frac{1}{n^{(1-2 c)}}\right)$.

Proof Consider the case where $\tau \lambda \notin\left\{w_{1}, w_{2}, \cdots, w_{q}\right\}$. It is clear that the variance of $f_{N}$ can be written as follows:

$$
\operatorname{var}\left[f_{N}(\lambda)\right]=\int_{R^{2}} W_{N}^{(1)}(\lambda-u) W_{N}^{(1)}\left(\lambda_{1}-u^{\prime}\right) \operatorname{cov}\left[\hat{I}_{N}(u), \hat{I}_{N}\left(u^{\prime}\right)\right] d u d u^{\prime}
$$

By using the fact that $W$ is zero for $|\lambda|>1$, for large $N$, we get

$$
\operatorname{var}\left[f_{N}(\lambda)\right]=\int_{-1}^{1} \operatorname{cov}\left[\hat{I}_{N}\left(\lambda-\frac{x_{1}}{M_{N}}\right), \hat{I}_{N}\left(\lambda-\frac{x_{1}^{\prime}}{M_{N}}\right)\right] W\left(x_{1}\right) W\left(x_{1}^{\prime}\right) d x_{1} d x_{1}^{\prime} .
$$

We define two subsets of the $[-1,1]^{2}$ by:

$$
\begin{array}{ll}
\text { - } L_{1}=\left\{\left(x_{1}, x_{1}^{\prime}\right) \in[-1,1]^{2} ; \quad\left|x_{1}-x_{1}^{\prime}\right|>\sigma_{N}\right\}, \\
\text { - } L_{2}=\left\{\left(x_{1}, x_{1}^{\prime}\right) \in[-1,1]^{2} ;\left|x_{1}-x_{1}^{\prime}\right| \leq \sigma_{N}\right\},
\end{array}
$$


where $\sigma_{N}$ is a nonnegative real, converging to 0 . We split the integral into an integral over the sub region $L_{2}$ and an integral over $L_{1}: \operatorname{var}\left[f_{N}(\lambda)\right]=\int_{L_{2}}+\int_{L_{1}}^{\stackrel{\Delta}{=}} J_{1}+J_{2}$.

By Cauchy-Schwartz inequality and theorem 3.1, we obtain

$$
\begin{gathered}
J_{1} \leq C \int_{\left|x_{1}-x_{1}^{\prime}\right| \leq \sigma_{N}} W\left(x_{1}\right) W\left(x_{1}^{\prime}\right) d x_{1} d x_{1}^{\prime} \text { where } C \text { is constant. Thus, we obtain } \\
J_{1}=O\left(\sigma_{N}\right)
\end{gathered}
$$

It remains to show that $J_{2}$ converges to zero. For simplicity, we define

$$
\lambda_{1}=\lambda-\frac{x_{1}}{M_{N}^{(1)}} ; \quad \lambda_{2}=\lambda-\frac{x_{1}^{\prime}}{M_{N}^{(1)}}, \quad \text { and } \quad C(\lambda)=\operatorname{cov}\left[\hat{I}_{N}\left(\lambda-\frac{x_{1}}{M_{N}^{(1)}}\right), \hat{I}_{N}\left(\lambda-\frac{x_{1}^{\prime}}{M_{N}^{(1)}}\right)\right]
$$

We first show that $C(\lambda)$ converges to zero uniformly in $x_{1}, x_{1}^{\prime} \in[-1,1]$. Indeed, from lemma 2.3 , we have

$$
E \hat{I}_{N}(v)-\hat{I}_{N}(v)=F_{p, \alpha}^{-1}\left[C_{\alpha}\right]^{-p / \alpha} \int_{-\infty}^{\infty} \frac{\operatorname{Re}\left(e^{i u I_{N}(v)}\right)-e^{-C_{\alpha}|u|^{\alpha} \psi_{N}(v)}}{|u|^{1+p}} d u
$$

Thus, the expression of the covariance becomes

$$
\begin{aligned}
C(\lambda)= & F_{p, \alpha}^{-2} C_{\alpha}^{-\frac{2 p}{\alpha}} \int_{R^{2}} E\left[\prod_{k=1}^{2} \cos \left(u_{k} I_{N}\left(\lambda_{k}\right)\right)\right] \\
& -\exp \left\{-C_{\alpha} \sum_{k=1}^{2}\left|u_{k}\right|^{\alpha} \psi_{N}\left(\lambda_{k}\right)\right\} \frac{d u_{1} d u_{2}}{\left|u_{1} u_{2}\right|^{1+p}}
\end{aligned}
$$

The following equality: $2 \cos x \cos y=\cos (x+y)+\cos (x-y)$, implies that

$$
\begin{aligned}
E\left[\prod_{k=1}^{2}\left(\cos u_{k} I_{N}\left(\lambda_{k}\right)\right)\right] & =\frac{1}{2} \exp \left[-C_{\alpha} \int\left|(\tau)^{\frac{1}{\alpha}} \sum_{k=1}^{2} u_{k} H_{N}\left(\tau \lambda_{k}-\tau \nu\right)\right|^{\alpha} d \mu(v)\right] \\
& +\frac{1}{2} \exp \left[-C_{\alpha} \int\left|(\tau)^{\frac{1}{\alpha}} \sum_{k=1}^{2}(-1)^{k+1} u_{k} H_{N}\left(\tau \lambda_{k}-\tau \nu\right)\right|^{\alpha} d \mu(v)\right] .
\end{aligned}
$$

By substituting the expression for $C(\lambda)$ and changing the variable $u_{2}$ to $\left(-u_{2}\right)$ in the second term, we obtain 


$$
\text { where } K=C_{\alpha} \int_{R}\left|(\tau)^{\frac{1}{\alpha}} \sum_{k=1}^{2} u_{k} H_{N}\left(\tau \lambda_{k}-\tau v\right)\right|^{\alpha} d \xi(v) \text { and }
$$

$$
K^{\prime}=C_{\alpha} \tau \sum_{k=1}^{2}\left|u_{k}\right|^{\alpha} \int_{R}\left|H_{N}\left(\tau \lambda_{k}-\tau v\right)\right|^{\alpha} \xi(v) d v
$$

Since $\quad K, K^{\prime}>0, \quad\left|e^{-K}-e^{-K^{\prime}}\right| \leq\left|K-K^{\prime}\right| \exp \left\{\left|K-K^{\prime}\right|-K^{\prime}\right\} \quad, \quad$ we $\quad$ obtain: $\left|K-K^{\prime}\right| \leq 2 C_{\alpha} \tau|u|^{\frac{\alpha}{2}} Q_{N}\left(\lambda_{1} ; \lambda_{2}\right)$,

$Q_{N}\left(\lambda_{1} ; \lambda_{2}\right)=\int_{-\Omega}^{\Omega}\left|H_{N}\left(\tau \lambda_{1}-\tau u\right)\right|^{\frac{\alpha}{2}}\left|H_{N}\left(\tau \lambda_{2}-\tau u\right)\right|^{\frac{\alpha}{2}} d \xi(u)$

Now, let us show that $Q_{N}\left(\lambda_{1} ; \lambda_{2}\right)$ converges to zero. Indeed, since $\phi$ is bounded on $[-\Omega, \Omega]$, we have

$$
\begin{aligned}
Q_{N}\left(\lambda_{1} ; \lambda_{2}\right) & \leq \sup (\phi) \int_{-\Omega}^{\Omega}\left|H_{N}\left(\tau \lambda_{1}-\tau u\right) H_{N}\left(\tau \lambda_{2}-\tau u\right)\right|^{\frac{\alpha}{2}} d u \\
& +\sum_{i=1}^{q} c_{i}\left|H_{N}\left(\tau \lambda_{1}-\tau w_{i}\right) H_{N}\left(\tau \lambda_{2}-\tau w_{i}\right)\right|^{\frac{\alpha}{2}}
\end{aligned}
$$

From the definition of $H_{N}$, we can write

$$
\int_{-\Omega}^{\Omega}\left|H_{N}\left(\tau \lambda_{1}-\tau v\right) H_{N}\left(\tau \lambda_{2}-\tau v\right)\right|^{\frac{\alpha}{2}} d v=\int_{-\Omega}^{\Omega} \frac{1}{B_{\alpha, N}^{\prime}}\left|\frac{\sin \left[\frac{n}{2}\left(\tau \lambda_{1}-\tau v\right)\right]}{\sin \left[\frac{1}{2}\left(\tau \lambda_{1}-\tau v\right)\right]}\right|^{k \alpha}\left|\frac{\sin \left[\frac{n}{2}\left(\tau \lambda_{2}-\tau v\right)\right]}{\sin \left[\frac{1}{2}\left(\tau \lambda_{2}-\tau v\right)\right]}\right| d v
$$

\section{a) First step:}

We show that the denominators of the first and second terms under the last integral do not vanish

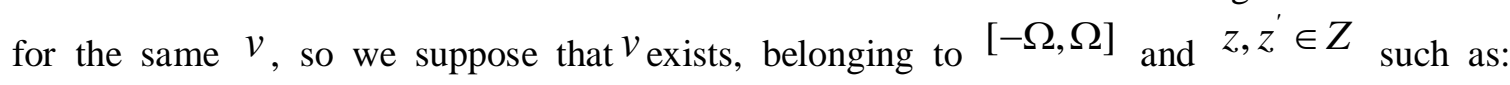
$\tau \lambda_{1}-\tau \nu=2 z \pi$ and $\tau \lambda_{2}-\tau \nu=2 z^{\prime} \pi$. Since $\lambda_{1} \not \lambda_{2}$, then $z$ and $z^{\prime}$ are different. Therefore, $\quad z-z^{\prime}=\frac{\tau}{2 \pi}\left(\lambda_{1}-\lambda_{2}\right)$. Hence, $\quad\left|z-z^{\prime}\right|=\frac{1}{w}\left|\lambda_{1}-\lambda_{2}\right| . \quad$ As $\quad \lim _{N \rightarrow \infty}\left|\lambda_{1}-\lambda_{2}\right|=0$, consequently, for a large $N$ we get: $|z-z|<\frac{1}{2}$.

Thus, we obtain a contradiction with the fact that $z$ and $z^{\prime}$ are different integers. 


\section{b) Second step}

We assume there exist $q$ points, $V_{1}, V_{2}, \cdots, V_{q} \in[-\Omega, \Omega]$ such as:

for $j=1,2, \cdots, q \quad \tau \lambda_{1}-\tau V_{j} \in 2 \pi Z$ then $\frac{\lambda_{1}}{w}-\frac{V_{j}}{w} \in Z$, and we assume that there exist $q^{\prime}$ points $V_{1}^{\prime}, V_{2}^{\prime}, \cdots, V_{q}^{\prime}, \in[-\Omega, \Omega]$ such that for $i=1,2, \cdots, q^{\prime} \quad \frac{\lambda_{2}}{w}-\frac{V_{i}^{\prime}}{w} \in Z$. Showing that, $\left|V_{j}\right| \neq \Omega, \quad\left|V_{i}^{\prime}\right| \neq \Omega \quad$ for $\quad 1 \leq j \leq q \quad$ and $\quad 1 \leq i \leq q^{\prime}$. Indeed, $\quad-1<\frac{\lambda-\Omega}{w}<0 \quad$ and $0<\frac{\lambda+\Omega}{w}<1$ because $w>2 \Omega$. Hence $\frac{\lambda-\Omega}{w} \notin Z$ and $\frac{\lambda+\Omega}{w} \notin Z$. For a large $N$, we get that $\left[\frac{\lambda-\Omega}{w}\right]_{E}<\frac{\lambda_{1}}{w}-\frac{\Omega}{w}<1+\left[\frac{\lambda-\Omega}{w}\right]_{E}, \quad$ where $[x]_{E}$ is the integer part of $x$. Hence, $\frac{\lambda_{1}}{w}-\frac{\Omega}{w} \notin Z$. In the same manner, we show that $\frac{\lambda_{1}}{w}+\frac{\Omega}{w} \notin Z$ $\frac{\lambda_{2}+\Omega}{w} \notin Z$. Thus, $\left|V_{j}\right| \neq \Omega$ and $\left|V_{i}^{\prime}\right| \neq \Omega$.

\section{c) Third step}

We classify $V_{j}$ and $V_{i}^{\prime}$ by increasing order: $-\Omega<V_{j_{1}}<V_{j_{2}}<\ldots<V_{j^{\prime}+q^{\prime}}<\Omega$, and we write the integral in the following manner:

$$
\int_{-\Omega}^{\Omega}\left|\frac{\sin \left[\frac{n}{2}\left(\tau \lambda_{1}-\tau \nu\right)\right]}{\sin \left[\frac{1}{2}\left(\tau \lambda_{1}-\tau \nu\right)\right]}\right|\left|\frac{\sin \left[\frac{n}{2}\left(\tau \lambda_{2}-\tau v\right)\right]}{\sin \left[\frac{1}{2}\left(\tau \lambda_{2}-\tau_{1} v\right)\right]}\right|^{k \alpha} d v=I_{1}+\sum_{i=1}^{q+q^{\prime}} I_{2, i}+\sum_{i=1}^{q+q^{\prime}-1} I_{3, i}+I_{4}
$$

where 


$$
\begin{aligned}
& I_{1}=\left.\int_{-\Omega_{1}}^{V_{j_{1}}-\delta(N)}\left|\frac{\sin \left[\frac{n}{2}\left(\tau \lambda_{1}-\tau v\right)\right]}{\sin \left[\frac{1}{2}\left(\tau \lambda_{1}-\tau v\right)\right]}\right| \frac{\sin \left[\frac{n}{2}\left(\tau \lambda_{2}-\tau v\right)\right]}{\sin \left[\frac{1}{2}\left(\tau \lambda_{2}-\tau v\right)\right]}\right|^{k \alpha} d v \\
& I_{2, i}=\left.\int_{V_{j_{i}}-\delta(N)}^{V_{j_{i}}+\delta(N)}\left|\frac{\sin \left[\frac{n}{2}\left(\tau \lambda_{1}-\tau \nu\right)\right]}{\sin \left[\frac{1}{2}\left(\tau \lambda_{1}-\tau \nu\right)\right]}\right| \frac{\sin \left[\frac{n}{2}\left(\tau \lambda_{2}-\tau v\right)\right]}{\sin \left[\frac{1}{2}\left(\tau \lambda_{2}-\tau v\right)\right]}\right|^{k \alpha} d v \\
& I_{3, i}=\int_{V_{j_{i}}+\delta(N)}^{V_{j_{i+1}}-\delta(N)}\left|\frac{\sin \left[\frac{n}{2}\left(\tau \lambda_{1}-\tau \nu\right)\right]}{\sin \left[\frac{1}{2}\left(\tau \lambda_{1}-\tau \nu\right)\right]}\right| \frac{\sin \left[\frac{n}{2}\left(\tau \lambda_{2}-\tau \nu\right)\right]}{\sin \left[\frac{1}{2}\left(\tau \lambda_{2}-\tau \nu\right)\right]}||^{k \alpha} d v \\
& I_{4}=\int_{V_{j},+\delta(N)}^{\Omega}\left|\frac{\sin \left[\frac{n}{2}\left(\tau \lambda_{1}-\tau \nu\right)\right]}{\sin \left[\frac{1}{2}\left(\tau \lambda_{1}-\tau \nu\right)\right]}\right| \frac{\sin \left[\frac{n}{2}\left(\tau \lambda_{2}-\tau \nu\right)\right]}{\sin \left[\frac{1}{2}\left(\tau_{1} \lambda_{2}-\tau \nu\right)\right]} \mid d v
\end{aligned}
$$

where $\delta(N)$ is a nonnegative real number converging to zero and satisfying:

$$
\begin{aligned}
& -\Omega<V_{j_{1}}-\delta(N)<V_{j_{1}}+\delta(N)<V_{j_{2}}-\delta(N)<V_{j_{2}}+\delta(N)<\ldots \ldots<V_{j_{q+q^{\prime}}}-\delta(N)<V_{q_{q+q^{\prime}}}+\delta(N)<\Omega, \\
& \text { and } \delta(N)<\left|\frac{\lambda_{1}-\lambda_{2}}{2}\right| .
\end{aligned}
$$

First, we show that the first integral converges to zero. We know that for a large $N$, we have $\lambda_{1}<\Omega$. For simplicity without loss of generality, we assume that for all $i, \tau \lambda_{1}-\tau V_{j_{i}}=2 k_{i} \pi$ with $k_{i} \in Z$. Similarly, we can show the result if we rather assume that $\tau \lambda_{2}-\tau V_{j_{i}}=2 k_{i} \pi$ with $k_{i} \in Z$.Since there is no $v$ between $-\Omega$ and $V_{j_{1}}-\delta(N)$ on which the denominators are vanishing,

$$
\begin{aligned}
& I_{1} \leq \frac{V_{j_{1}}-\delta(N)+\Omega}{\inf \left[\left|\sin \frac{\tau \delta(N)}{2}\right|^{k \alpha},\left|\sin \frac{\tau\left(\lambda_{1}+\Omega\right)}{2}\right|^{k \alpha}\right] \inf \left[\left|\sin \frac{\tau\left(\lambda_{2}-V_{j_{1}}+\delta(N)\right)}{2}\right|^{k \alpha},\left|\sin \frac{\tau\left(\lambda_{2}+\Omega\right)}{2}\right|^{k \alpha}\right]} . \\
& \left|\sin \frac{\tau\left(\lambda_{2}-V_{j_{1}}+\delta(N)\right)}{2}\right|^{k \alpha}=\left|\sin \frac{\tau \mid \lambda_{2}-\lambda_{1}+\delta(N)}{2}\right|^{k \alpha} \text {. For a large } N \text {, we have }
\end{aligned}
$$


$\frac{\tau\left|\lambda_{2}-\lambda_{1}+\delta(N)\right|}{2} \leq \tau \Omega+\frac{\tau \delta(N)}{2}<\pi-\frac{\tau \delta(N)}{2}$

On the other hand, two cases are possible:

1. $\lambda_{2}-\lambda_{1}>0$, then we have $\left|\lambda_{2}-\lambda_{1}+\delta(N)\right|=\lambda_{2}-\lambda_{1}+\delta(N)>\delta(N)$

2. $\lambda_{2}-\lambda_{1}<0$, since $\left|\lambda_{2}-\lambda_{1}\right|>2 \delta(N)$, we have

$\left|\lambda_{2}-\lambda_{1}+\delta(N)\right|=\lambda_{1}-\lambda_{2}-\delta(N)>\delta(N)$

Therefore, $\quad \frac{\tau \delta(N)}{2}<\frac{\tau\left|\lambda_{2}-\lambda_{1}+\delta(N)\right|}{2}<\pi-\frac{\tau \delta(N)}{2}$. For a large $N$, we have $\tau \delta(N)<2 \pi-\tau\left(\lambda_{1}+\Omega\right)$ and $\tau \delta(N)<2 \pi-\tau\left(\lambda_{2}+\Omega\right)$. Then,

$$
\begin{aligned}
\frac{\tau \delta(N)}{2} & <\frac{\tau\left(\lambda_{1}+\Omega\right)}{2}<\pi-\frac{\tau \delta(N)}{2} \text { and } \frac{\tau \delta(N)}{2}<\frac{\tau\left(\lambda_{2}+\Omega\right)}{2}<\pi-\frac{\tau \delta(N)}{2} \text {. Consequently, } \\
I_{1} & \leq \frac{V_{j_{1}}-\delta(N)+\Omega}{\left|\sin \frac{\tau \delta(N)}{2}\right|^{2 k \alpha}} .
\end{aligned}
$$

For the integral $I_{2, i}$, we bound the first fraction under integral by $n^{k \alpha}$ :

$$
\begin{aligned}
& I_{2, i} \leq n^{k \alpha} \int_{V_{j_{i}}-\delta(N)}^{V_{j_{i}}+\delta(N)} \frac{1}{\left|\sin \left[\frac{1}{2}\left(\tau \lambda_{2}-\tau v\right)\right]\right|^{k \alpha}} d v \\
& \text { putting } v=u-\frac{2 k \pi}{\tau} \text {, we get substituting for } V_{j_{i}} \text { in the last inequality and }
\end{aligned}
$$$$
I_{2, i} \leq n^{k \alpha} \int_{\lambda_{1}-\delta(N)}^{\lambda_{1}+\delta(N)} \frac{1}{\left|\sin \left[\frac{1}{2}\left(\tau \lambda_{2}-\tau u\right)\right]\right|^{k \alpha}} d u
$$$$
\left|\lambda_{2}-u\right| \geq\left|\lambda_{2}-\lambda_{1}\right|-\left|\lambda_{1}-u\right| \geq\left|\lambda_{2}-\lambda_{1}\right|-\delta(N)>\frac{\left|\lambda_{2}-\lambda_{1}\right|}{2}
$$

Since $\delta(N)$ converges to zero, for a large $N$ we have $\delta(N)<\frac{2}{\tau}\left(\pi-\frac{\tau}{2}\left|\lambda_{2}-\lambda_{1}\right|\right)$, therefore $0<\tau \frac{\left|\lambda_{2}-\lambda_{1}\right|}{4}<\tau \frac{\left|\lambda_{2}-u\right|}{2}<\tau \frac{\left|\lambda_{2}-\lambda_{1}\right|+\delta(N)}{2}<\pi$.

Consequently: 


$$
I_{2, i} \leq \frac{2 \delta(N) n^{k \alpha}}{\inf \left[\sin \frac{\left|\lambda_{1}-\lambda_{2}\right| \tau}{4} ; \sin \frac{\left|\lambda_{1}-\lambda_{2}\right| \tau+\delta(N) \tau}{2}\right]}
$$

where $\left|\lambda_{2}-\lambda_{1}\right|=\left|\frac{x_{2}-x_{1}}{M_{N}}\right|$. Then, for a large $N$, we have $\frac{3 \tau}{2}\left|\lambda_{2}-\lambda_{1}\right|<2 \pi$. $\tau \frac{\left|\lambda_{2}-\lambda_{1}\right|}{4}<\frac{\tau\left|\lambda_{2}-\lambda_{1}\right|+\tau \delta(N)}{2}<\pi-\tau \frac{\left|\lambda_{2}-\lambda_{1}\right|}{4}$.

$$
I_{2, i} \leq \frac{2 \delta(N) n^{k \alpha}}{\left|\sin \frac{\left.\tau\left|\lambda_{2}-\lambda_{1}\right|\right)}{4}\right|^{k \alpha}}
$$

Since there is no $v$ between $V_{j_{i}}+\delta(N)$ and $V_{j_{i+1}}-\delta(N)$ on which the denominators are vanishing, we get:

$$
I_{3, i} \leq \frac{V_{j_{i+1}}-V_{j_{i}}-2 \delta(N)}{A \times B},
$$

where

$$
\begin{aligned}
& A=\inf \left(\left|\sin \frac{\tau \lambda_{1}-\tau V_{j_{i}}-\tau \delta(N)}{2}\right|^{k \alpha},\left|\sin \frac{\tau \lambda_{1}-\tau V_{j_{i+1}}+\tau \delta(N)}{2}\right|^{k \alpha}\right) \\
& B=\inf \left(\left|\sin \frac{\tau \lambda_{2}-\tau V_{j_{i}}-\tau \delta(N)}{2}\right|^{k \alpha},\left|\sin \frac{\tau \lambda_{2}-\tau V_{j_{i+1}}+\tau \delta(N)}{2}\right|^{k \alpha}\right)
\end{aligned}
$$

It follows from the hypothesis on $\delta(N)$ that

$$
\frac{\tau \delta(N)}{2}<\frac{\tau\left|\lambda_{1}-\lambda_{2}\right|}{2}-\frac{\tau|\delta(N)|}{2}<\frac{\tau\left|\lambda_{1}-\lambda_{2}-\delta(N)\right|}{2}<\frac{\tau\left|\lambda_{1}-\lambda_{2}\right|}{2}+\frac{\tau|\delta(N)|}{2}<\pi-\frac{\tau \delta(N)}{2} .
$$

Thus, by using the definition of $V_{j_{i}}$, we obtain 
$\frac{1}{\left|\sin \frac{\tau \lambda_{2}-\tau V_{j_{i}}-\tau \delta(N)}{2}\right|^{k \alpha}}<\frac{1}{\left|\sin \frac{\tau \delta(N)}{2}\right|^{k \alpha}}$.

We use the same way for bounding the other terms

$$
I_{3, i} \leq \frac{V_{j_{i+1}}-V_{j_{i}}-2 \delta(N)}{\left|\sin \frac{\tau \delta(N)}{2}\right|^{2 k \alpha}}
$$

Similarly, since there is no $v$ between $V_{j_{q+q^{\prime}}}+\delta(N)$

and we get :
and $\Omega$ which the denominators are vanishing, we can show that:

$$
\begin{aligned}
& I_{4} \leq \frac{V_{j_{i+1}}-V_{j_{i}}-2 \delta(N)}{E \times F}, \\
& E=\operatorname{whf}\left(\left|\sin \frac{\tau \lambda_{1}-\tau \Omega}{2}\right|^{k \alpha},\left|\sin \frac{\tau \lambda_{1}-\tau V_{j_{q+q^{\prime}}}-\tau \delta(N)}{2}\right|^{k \alpha}\right) \\
& F=\inf \left(\left|\sin \frac{\tau \lambda_{2}-\tau \Omega}{2}\right|^{k \alpha},\left|\sin \frac{\tau \lambda_{2}-\tau V_{j_{q+q^{\prime}}}-\tau \delta(N)}{2}\right|^{k \alpha}\right)
\end{aligned}
$$

Since $\delta(N)$ converges to zero, for a large $N$, we have $\delta(N)<\frac{2}{\tau}\left(\pi-\frac{\tau}{2}\left|\lambda_{1}-\Omega\right|\right)$, and

$$
\delta(N)<\frac{2}{\tau}\left(\pi-\frac{\tau}{2}\left|\lambda_{1}-\lambda_{2}\right|\right) \text {. It follows that: } I_{4} \leq \frac{\Omega-V_{j_{q+q^{\prime}}}-\delta(N)}{\left|\sin \frac{\tau \delta(N)}{2}\right|^{2 k \alpha} .}
$$

previous increases, we obtain

We recapitulate, from the

$$
\begin{aligned}
& \int_{-\Omega}^{\Omega}\left|\frac{\sin \left[\frac{n}{2}\left(\tau \lambda_{1}-\tau \nu\right)\right]}{\sin \left[\frac{1}{2}\left(\tau \lambda_{1}-\tau v\right)\right]}\right|\left|\frac{\sin \left[\frac{n}{2}\left(\tau \lambda_{2}-\tau \nu\right)\right]}{\sin \left[\frac{1}{2}\left(\tau \lambda_{2}-\tau \nu\right)\right]}\right| \quad d v \leq \frac{\Omega+V_{j_{1}}-\delta(N)}{\left|\sin \frac{\tau \delta(N)}{2}\right|^{k \alpha \alpha}}+\sum_{i=1}^{q+q^{\prime}} \frac{2 n^{k \alpha} \delta(N)}{\left|\sin \frac{\tau\left|\lambda_{2}-\lambda_{1}\right|}{4}\right|^{k \alpha}} \\
& +\sum_{i=1}^{q+q^{\prime}-1} \frac{V_{j_{i+1}}-V_{j_{i}}-\delta(N)}{\left|\sin \frac{\tau \delta(N)}{2}\right|^{2 k \alpha}}+\frac{\Omega-V_{j_{q+q^{\prime}}}-\delta(N)}{\left|\sin \frac{\tau \delta(N)}{2}\right|^{2 k \alpha}}
\end{aligned}
$$

After simplification, we have 
$\int_{-\Omega}^{\Omega}\left|\frac{\sin \left[\frac{n}{2}\left(\tau \lambda_{1}-\tau v\right)\right]}{\sin \left[\frac{1}{2}\left(\tau \lambda_{1}-\tau v\right)\right]}\right| \frac{\sin \left[\frac{n}{2}\left(\tau \lambda_{2}-\tau v\right)\right]}{\sin \left[\frac{1}{2}\left(\tau \lambda_{2}-\tau v\right)\right]} \mid \quad d v \leq \frac{2 \Omega-2\left(q+q^{\prime}+1\right) \delta(N)}{\left|\sin \frac{\tau \delta(N)}{2}\right|^{2 k \alpha}}+\frac{2 n^{k \alpha} \delta(N)\left(q+q^{\prime}\right)}{\left|\sin \frac{\tau\left|\lambda_{2}-\lambda_{1}\right|}{4}\right|^{k \alpha}}$.

By bounding the first term on the right hand side of the last inequality and using the following inequality $\left|\sin \frac{x}{2}\right| \geq \frac{|x|}{\pi}$, we get $\int_{-\Omega}^{\Omega}\left|\frac{\sin \left[\frac{n}{2}\left(\tau \lambda_{1}-\tau v\right)\right]}{\sin \left[\frac{1}{2}\left(\tau \lambda_{1}-\tau \nu\right)\right]}\right|\left|\frac{\sin \left[\frac{n}{2}\left(\tau \lambda_{2}-\tau \nu\right)\right]}{\sin \left[\frac{1}{2}\left(\tau \lambda_{2}-\tau \nu\right)\right]}\right| \quad d v \leq \frac{2 \Omega \pi^{2 k \alpha}}{(\tau \delta(N))^{2 k \alpha}}+\frac{2 n^{k \alpha} \delta(N)\left(q+q^{\prime}\right)(2 \pi)^{k \alpha}}{\left(\frac{\tau\left|x_{2}-x_{1}\right|}{M_{N}}\right)^{k \alpha}}$.

It follows from the lemma 2.1

$$
\begin{aligned}
\frac{1}{B_{\alpha, N}^{\prime}} \int_{-\Omega}^{\Omega}\left|\frac{\sin \left[\frac{n}{2}\left(\tau \lambda_{1}-\tau \nu\right)\right]}{\sin \left[\frac{1}{2}\left(\tau \lambda_{1}-\tau \nu\right)\right]}\right|\left|\frac{\sin \left[\frac{n}{2}\left(\tau \lambda_{2}-\tau \nu\right)\right]}{\sin \left[\frac{1}{2}\left(\tau \lambda_{2}-\tau \nu\right)\right]}\right| d v \leq \\
\frac{1}{\pi}\left(\frac{\pi}{2}\right)^{2 k \alpha}\left(\frac{2 \Omega \pi^{2 k \alpha}}{n^{2 k \alpha-1}(\tau \delta(N))^{2 k \alpha}}+\frac{2 \delta(N)\left(q+q^{\prime}\right)(2 \pi)^{k \alpha}}{n^{k \alpha-1}\left(\frac{\tau \sigma_{N}}{M_{N}}\right)^{k \alpha}}\right) .
\end{aligned}
$$

In order to obtain the convergence of the last expression to zero, we choose $\delta(N)=n^{-\beta}, \quad \beta>0$, such as

$$
\lim _{n \rightarrow \infty} \frac{n^{2 k \alpha \beta}}{n^{2 k \alpha-1}}=0 \quad \text { and } \quad \lim _{n \rightarrow \infty} \frac{1}{n^{\beta+k \alpha-1}\left(\frac{\sigma_{N}}{M_{N}}\right)^{k \alpha}}=0
$$

Thus, from (18) $Q_{N}$ converges to zero. On the other hand,

$$
\begin{aligned}
& C(\lambda) \leq F_{p, \alpha}^{-2} C_{\alpha}^{-\frac{2 p}{\alpha}} \int_{-\infty}^{\infty} \int_{-\infty}^{\infty}\left|K-K^{\prime}\right| e^{\left|K-K^{\prime}\right|-K^{\prime}} \frac{d u_{1} d u_{2}}{\left|u_{1} u_{2}\right|^{1+p}}, \\
& \text { where }\left|K-K^{\prime}\right|-K^{\prime} \leq-C_{\alpha} \sum_{k^{\prime}=1}^{2}\left|u_{k^{\prime}}\right|^{\alpha}\left|\psi_{N}\left(\lambda_{k^{\prime}}\right)-\tau Q_{N}\left(\lambda_{1} ; \lambda_{2}\right)\right| .
\end{aligned}
$$


We denote by : $\delta_{\left(N, k^{\prime}\right)}=\psi_{N}\left(\lambda_{k^{\prime}}\right)-\tau Q_{N}\left(\lambda_{1} ; \lambda_{2}\right)$. It follows from (18) and (20) that $\delta_{\left(N, k^{\prime}\right)}$ converges to $\phi(\lambda)$. Hence,

$$
C(\lambda) \leq F_{p, a}^{-2} C_{\alpha}^{-\frac{2 p}{\alpha}} 2 \tau C_{\alpha} Q_{N}\left(\lambda_{1} ; \lambda_{2}\right) 4 \prod_{k^{\prime}=1}^{2} \int_{0}^{\infty} \exp \left[-C_{\alpha}\left(u_{k^{\prime}}\right)^{\alpha}\left|\delta_{\left(N, k^{\prime}\right)}\right|\right] \frac{d u_{k^{\prime}}}{\left(u_{k^{\prime}}\right)^{1+p-\frac{\alpha}{2}}} .
$$

Putting $\quad u_{k^{\prime}}\left(\delta_{N, k^{\prime}}\right)^{\frac{1}{\alpha}}=v$, we obtain

$$
C(\lambda) \leq F_{p, \alpha}^{-2} C_{\alpha}^{-\frac{2 p}{\alpha}} 2 \tau C_{\alpha} \frac{Q_{N}\left(\lambda_{1} ; \lambda_{2}\right)}{\left[\delta_{(N, 1)} \delta_{(N, 2)}\right]^{\frac{1}{2}-\frac{p}{\alpha}}}\left(\int_{-\infty}^{\infty} \frac{e^{-C_{\alpha}|\nu|^{\alpha}}}{|v|^{1+p-\frac{\alpha}{2}}} d v\right)^{2} .
$$

Since $\phi(\lambda)>0, C(\lambda)$ converges uniformly in $x_{1}, x_{1}^{\prime} \in[-1,1]$ to zero. From (20), we obtain $J_{2}=O\left(\frac{1}{n^{2 k \alpha(1-\beta)-1}}+\frac{1}{n^{\beta+k \alpha-1}\left(\frac{\sigma_{N}}{M_{N}}\right)^{k \alpha}}\right)_{\text {Thus, }} \operatorname{var}\left[f_{N}(\lambda)\right]$ converges to zero and then, $f_{N}(\lambda)$ is an asymptotically unbiased and consistent estimator.

\section{Conclusions}

In this paper, we estimate the spectral density of mixed stable process with continuous time when the process is observed at discrete instants. The aliasing phenomenon is avoided by assuming that the spectral is a compact support. This work can be applied in various fields. For example:

- The study of soil cracking where the observed signal is the resistance of the soil. This resistance can have random jumps that are due to the presence of some stones. Thus, the spectral measurement will be composed of two parts, one continuous and the other discrete corresponding to the resistance jumps encountered during the measurement.

- The growth of fruit on a tree can be considered as a continuous distribution, and when there is a fall of a fruit, the other fruits remaining on the tree absorb more energy and their growth will have a jump in value.

The perspective of this work is to optimize the smoothing parameters to have a better rate of convergence. For this purpose, the cross-validation method will be the most appropriate tool.

This work can also be completed by considering a more general case when we observe the process with random errors. In this case, we will use the deconvolution methods, which have proved their efficiency in the presence of random errors. 
It would be interesting to give an estimator of the mode of the spectral density representing the frequency where the spectral density reaches the maximum of energy. For that, we must estimate the derivative of the spectral density function.

\section{REFERENCES}

[1] S. Cambanis, (1983)“Complex symetric stable variables and processes” In P.K.SEN, ed, Contributions to Statistics”: Essays in Honour of Norman L. Johnson North-Holland. New York,(P. K. Sen. ed.), pp. 63-79

[2] S. Cambanis, and M. Maejima (1989). "Two classes of self-similar stable processes with stationary increments". Stochastic Process. Appl. Vol. 32, pp. 305-329

[3] M.B. Marcus and K. Shen (1989). "Bounds for the expected number of level crossings of certain harmonizable infinitely divisible processes". Stochastic Process. Appl., Vol. 76, no. 1 pp 1-32.

[4] E. Masry, S. Cambanis (1984). "Spectral density estimation for stationary stable processes". Stochastic processes and their applications, Vol. 18, pp. 1-31 North-Holland.

[5] G. Samorodnitsky and M. Taqqu (1994). "Stable non Gaussian processes ». Chapman and Hall, New York.

[6] K., Panki and S. Renming (2014). "Stable process with singular drift”. Stochastic Process. Appl., Vol. 124, no. 7, pp. 2479-2516

[7] C. Zhen-Qing and W. Longmin (2016). "Uniqueness of stable processes with drift." Proc. Amer. Math. Soc., Vol. 144, pp. 2661-2675

[8] K. Panki, K. Takumagai and W. Jiang (2017). "Laws of the iterated logarithm for symmetric jump processes". Bernoulli, Vol. 23, n 4 pp. 2330-2379.

[9] M. Schilder (1970). "Some Structure Theorems for the Symmetric Stable Laws". Ann. Math. Statist., Vol. 41, no. 2, pp. 412-421.

[10] R. Sabre (2012b). "Missing Observations and Evolutionary Spectrum for Random". International Journal of Future Generation Communication and Networking, Vol. 5, n 4, pp. 55-64.

[11] E. Sousa (1992). "Performance of a spread spectrum packet radio network link in a Poisson of interferences". IEEE Trans. Inform. Theory, Vol. 38, pp. 1743-1754

[12] M. Shao and C.L. Nikias (1993). "Signal processing with fractional lower order moments: Stable processes and their applications", Proc. IEEE, Vol.81, pp. 986-1010

[13] C.L. Nikias and M. Shao (1995). "Signal Processing with Alpha-Stable Distributions and Applications". Wiley, New York

[14] S. Kogon and D. Manolakis (1996). "Signal modeling with self-similar alpha- stable processes: The fractional levy motion model”. IEEE Trans. Signal Processing, Vol 44, pp. 1006-1010

[15] N. Azzaoui, L. Clavier, R. Sabre, (2002). "Path delay model based on stable distribution for the 60GHz indoor channel” IEEE GLOBECOM, IEEE, pp. 441-467

[16] J.P. Montillet and Yu. Kegen (2015). "Modeling Geodetic Processes with Levy alpha-Stable Distribution and FARIMA", Mathematical Geosciences. Vol. 47, no. 6, pp. 627-646.

[17] M. Pereyra and H. Batalia (2012). "Modeling Ultrasound Echoes in Skin Tissues Using Symmetric alpha-Stable Processes". IEEE Transactions on Ultrasonics, Ferroelectrics, and Frequency Control, Vol. 59, $\mathrm{n}^{\circ}$. 1, pp. 60-72.

[18] X. Zhong and A.B. Premkumar (2012). "Particle filtering for acoustic source tracking in impulsive noise with alpha-stable process". IEEE Sensors Journal, Vol. 13, no. 2, pp. 589 - 600.

[19] Wu. Ligang and W. Zidong (2015). "Filtering and Control for Classes of Two-Dimensional Systems". The series Studies in Systems of, Decision and Control, Vol.18, pp. 1-29.

[20] N. Demesh (1988). "Application of the polynomial kernels to the estimation of the spectra of discrete stable stationary processes”. (Russian) Akad.Nauk.ukrain. S.S.R. Inst.Mat. Preprint 64, pp. 12-36

[21] F. Brice, F. Pene, and M. Wendler, (2017) "Stable limit theorem for U-statistic processes indexed by a random walk", Electron. Commun. Prob., Vol. 22, no. 9, pp.12-20.

[21] R. Sabre (2019). “Alpha Stable Filter and Distance for Multifocus Image Fusion”. IJSPS, Vol. 7, no. 2, pp. 66-72.

[22] JN. Chen, J.C. Coquille, J.P. Douzals, R. Sabre (1997). "Frequency composition of traction and tillage forces on a mole plough". Soil and Tillage Research, Vol. 44, pp. 67-76.

[23] R. Sabre (1995). "Spectral density estimate for stationary symmetric stable random field", Applicationes Mathematicaes, Vol. 23, n. 2, pp. 107-133 
[24] R. Sabre (2012a). "Spectral density estimate for alpha-stable p-adic processes". Revisita Statistica, Vol. 72, n. 4, pp. 432-448.

[25] R. Sabre (2017). "Estimation of additive error in mixed spectra for stable prossess". Revisita Statistica, Vol. LXXVII, ${ }^{\circ}$. 2, pp. 75-90.

[26] E. Masry, (1978). "Alias-free sampling: An alternative conceptualization and its applications", IEEE Trans. Information theory, Vol. 24, pp.317-324.

\section{AUTHOR}

Rachid Sabre received the PhD degree in statistics from the University of Rouen, France, in 1993 and Habilitation to direct research (HdR) from the University of Burgundy, Dijon, France, in 2003. He joined Agrosup Dijon, France, in 1995, where he is an Associate Professor. From 1998 through 2010, he served as a member of Institut de Mathématiques de Bourgogne, France. He was a member of the Scientific Council AgroSup Dijon from 2009 to 2013. From 2012 to 2019, he has been a member of Laboratoire Electronic, Informatique, and Image (LE2I), France. Since 2019 he has been a member of Laboratory Biogeosciences UMR CNRS University Burgundy. He is author/co-author of numerous papers in scientific and technical journals and conference proceedings. His research interests lie in areas of statistical process and spectral analysis for signal and image processing.

(C) 2021 By AIRCC Publishing Corporation. This article is published under the Creative Commons Attribution (CC BY) license. 